\title{
Off-Line Routing with Small Queues on a Mesh-connected Processor Array
}

\author{
Danny Krizanc \\ Lata Narayanan \\ Department of Computer Science \\ University of Rochester \\ Rochester, NY 14627
}

\begin{abstract}
We present several efficient and simple algorithms for off-line routing that use very small queues. On an $n \times n$ mesh-connected processor array, we show a $2.5 n$ step algorithm to off-line route permutations using queues of size 2 , and a $2.25 n+3$ step algorithm that uses queues of size 4 . Our main result is that there exists an off-line algorithm for permutation routing on the $n \times n$ mesh-connected processor array, that takes $2.2 n+5$ steps, and uses queues of size not more than 14 . Our algorithms use novel and interesting techniques, and the bounds on the queue size are smaller than those of known algorithms with the same time complexity.
\end{abstract}

Keywords: Parallel algorithms, off-line routing, mesh-connected array.

\section{Introduction}

One of the key issues in the study of parallel computation is communication between processors. In the fixed connection network model, we isolate this into the problem of permutation routing. The permutation routing problem is specified by a network, and a set of packets, each with an origin and a destination, where the set of origin and destination pairs is constrained to form a permutation of the nodes of the network. A routing strategy must, for every permutation, find paths for each packet to move from its source to its destination, as well as specify when each packet crosses every edge in its path. Most work in this area has focused on trying to solve this problem in an on-line manner. In practice, however, in solving a particular problem, it is often the case that the same communication patterns have to be realized over and over again. Indeed, these patterns are known at the time of the routing algorithm's design. This motivates the problem of off-line routing. Once again, the routing strategy must find routes for all the packets, but the routes may now be calculated 
off-line. The running time of the algorithm is simply the time it takes for the every packet to reach its destination. The study of off-line routing is important not only because of its practical significance, but because it helps in the understanding of wherein the difficulty of the routing problem lies.

In this paper, we study the problem of off-line routing on the two-dimensional mesh-connected processor array. The mesh network is the basis for a number of proposed and implemented parallel computers. The simplicity and the regularity of its interconnection pattern make it ideal for VLSI implementation. Additionally, a number of important problems lend themselves to elegant and simple solutions on the mesh. Our model of the $n \times n$ mesh is sometimes referred to as the MIMD model.

In designing routing algorithms for the mesh, we are interested in minimizing both the routing time, and the maximum queue size at any processor. The diameter of the mesh immediately implies a lower bound of $2 n-2$ steps for the routing time, since in the worst case a permutation may have a packet originating in the top left corner processor and destined for the bottom right corner processor. Leighton, Makedon and Tollis [LMT89] gave an algorithm for on-line permutation routing (and therefore for off-line as well) which achieves this bound. However, in their algorithm the processors must store a large number of packets between communication steps. (The number is constant but estimated to be in excess of 500.) Recently, the bound on the queue size was improved to 58 [R091].

As a corollary to one of their theorems, Annexstein and Blaumslag [AB90] show how to route off-line in $3 n$ steps with at most 1 packet per processor. In this paper, we extend this result by showing that by allowing only a slight increase in the amount of storage required, a great improvement in the time can be made. We present a succession of three algorithms, each one working faster than the previous one. Our first algorithm routes permutations off-line in $2.5 n$ steps, using queues of size 2. The second algorithm solves the same problem in $2.25 n+3$ steps, and uses queues of size 4 . Finally, we show an algorithm that routes any permutation off-line in $2.2 n+5$ steps, using at most 14 packets per processor per step. Compare this with the result of Kunde [Kun88] that requires $c$ sized queues for an algorithm that uses $2 n+O(n / c)$ steps. For the case of the algorithm running in $2.2 n$ steps, Kunde's algorithm would require queues of size 30 . Additionally, our algorithm is conceptually simple, and uses new and elegant techniques. We also present ideas for how to route upto four permutations simulatneously and efficiently on the mesh. Furthermore, the techniques might extend to provide an off-line algorithm that runs in $2 n$ steps with very small queues.

The next section contains definitions relevant to our problem. Section 3 introduces some preliminaries and develops some basic techniques that we will require later. In Section 4, we present our off-line algorithms for routing. We conclude with some discussion and open problems. 


\section{Definitions}

The $n \times n$ mesh-connected array of processors (or two-dimensional mesh) contains $N=n^{2}$ processors arranged in two-dimensional grid without wrap-around edges. More precisely, it corresponds to the graph, $G=(V, E)$, with $V=\{(x, y) \mid x, y \in$ $\langle n\rangle\}$ and $E=\{((x, y),(x, y+1)) \mid x \in\langle n\rangle, y \in\langle n-1\rangle>\} \cup\{((x, y),(x+1, y)) \mid x \in$ $\langle n-1\rangle, y \in\langle n\rangle\}$, where $\langle n\rangle=\{1, \ldots, n\}$. The $d$-dimensional mesh is the logical extension of the two-dimensional version to higher dimensions.

The off-line routing problem is specified by a network $G$ and a set $P$ of pairs of nodes such that $\left(o_{x}, d_{x}\right)$ represents the origin and destination of packet $x$. The problem is to find a connected path along edges of $G$ from $o_{x}$ to $d_{x}$ for every $x$, as well as a schedule for when $x$ crosses every edge in its path. The packets are constrained to travel at most one edge in a time step. At most one packet can traverse an edge in any one time step. The time taken by the algorithm is the length of the longest schedule. The queue-size is the maximum number of packets that may be at any processor in a single time step during the course of the routing.

We will define a $(h, k)$ mapping to be a routing problem where not more than $h$ packets may originate at any given node and not more than $k$ packets can be destined for any node.

\section{Preliminary Results about Routing}

In this section, we introduce techniques and observations about the mesh that will be useful in constructing our final algorithm.

Lemma 1 On an $n$ processor linear array, any permutation can be routed (on- or off-line) in $n$ steps with at most 1 packet per processor per time step.

Proof. We use the well-known reduction of routing to sorting by destination. The odd-even transposition sort [TK77] achieves the above bound.

Lemma 2 On an $n$ processor linear array, 2 permutations can be simultaneously routed (on-or off-line) in $n+1$ steps with at most 2 packets per processor per time step.

Proof. Recall the way in which the odd-even transposition sort works. During odd steps of the sort, packets are compared and exchanged along odd edges of the array, and during the even steps along even edges. To perform 2 sorts simultaneously, start a second sort after the first step of the first sort using the odd edges while the first sort uses the even edges and vice versa. The second sort completes after $n+1$ steps. On any step a processor receives at most 2 packets (one belonging to each sort), so that at most 2 packets reside at a processor between steps. 
Lemma 3 On an $n \times n$ mesh, 2 permutations can be simultaneously routed off-line in $3 n$ steps with at most 2 packets per processor per time step.

Proof. Recall how Annexstein and Blaumslag [AB90] perform off-line permutation routing on the mesh. They show that any permutation can be realized by first performing a permutation of each of the rows, followed by a permutation of the columns and finishing with another permutation of the rows. (The three permutations are calculated off-line.) We observe that a second permutation may be simultaneously routed by first performing a permutation of each of the columns (while the first permutation is being routed in the rows) followed by permutations in the rows and then again in the columns. Using lemma 1, the above can be performed in $3 n$ steps. On any step, a processor receives at most 2 packets, so that at most 2 packets reside at a processor between steps.

Lemma 4 On an $n \times n$ mesh, 4 permutations can be simultaneously routed off-line in $3 n+3$ steps with at most 4 packets per processor per time step.

Proof. Analogous to lemma 3, we send 2 permutations first by row, then by column and finally by row and send the other 2 permutations first by column, then by row and finally by column. Using lemma 2 , the routing is completed in $3 n+3$ steps. On any step a processor receives at most 4 packets, so that at most 4 packets reside at a processor between steps.

Lemma 5 Consider an n-node linear array, where each processor holds a packet. All the packets have distinct destinations. Call these the permuting packets. At time step $i$, a packet with destination $n-i+1$ is generated at the first processor, and a packet with destination $i$ is generated at the processor labeled $n$. All these packets can be routed in $n$ steps, with at most 5 packets at any node.

Proof. Route packets using a farthest first strategy. Clearly, each of these three sets of packets can be routed in $n$ steps separately. Packets that are being generated at processor 1 do not interfere with the routing of packets that are generated at processor $n$. It suffices to analyze the packets moving in one direction, say downwards. The permuting packets are always ahead of packets that are being generated at processor 1 , and therefore never interfere with them. Each processor is the destination of 3 packets, and in every step receives at most 2 packets. Therefore, the queue size is bounded by 5 packets.

Consider an $n$-node linear array to be $k$ sub-arrays of size $n / k$ each. Let all the packets in sub-array $i$ be destined for distinct nodes inside sub-array $i$. Further let each packet in sub-array $i-1$ and $i+1$ be destined for the corresponding node in sub-array $i$. Lemma 5 tells us that if we route all packets greedily, then all packets can be routed in $n / k$ steps. In other words, packets can flow into a sub-array at the same time as packets are being permuted within the sub-array. It is easy to show 
similarly, that packets can move out of a sub-array at the same time as packets are being permuted in the sub-array. Note however, that moving packets in as well as moving packets out at the same time as permuting packets within a sub-array types of packets requires at least $3 n / 2 k$ steps, because at least $3 n / 2 k$ packets cross the middle edge in each sub-array.

\section{Algorithms for Off-line Routing}

We now use some of the techniques developed in the previous section to design efficient algorithms for off-line permutation routing with small queues. We begin with some definitions. Consider the division of the $n \times n$ mesh into sub-meshes, each of side $n / k$, where $k$ is an integer that divides $n$. The sub-meshes can be regarded as forming a $k \times k$ mesh, which will be referred to as the super-mesh. For a particular division of the mesh (that is, for fixed $k$ ), the following terms are uniquely defined.

\section{Definition 1 Intermediate sub-mesh}

For a given packet with source $(a, b)$ and destination $(c, d), 1 \leq a, b, c, d \leq n$, its intermediate sub-mesh is the unique sub-mesh that contains the processor labeled $(c, b)$.

\section{Definition 2 Virtual source (destination)}

The processor that corresponds to the source (destination) of a given packet in its intermediate sub-mesh is called its virtual source (destination). (Imagine overlaying the sub-mesh containing the actual source (destination) of the packet with its intermediate sub-mesh.)

\section{Definition 3 Routing sub-problem}

For each pair of sub-meshes, the set of packets originating in the first sub-mesh and destined for a processor in the second sub-mesh constitutes a routing subproblem. Therefore, there are $k^{2}$ routing sub-problems.

Theorem 1 On an $n \times n$ mesh, any permutation can be off-line routed in $2.5 n$ steps with at most 2 packets per processor per time step.

Proof. Divide the mesh into $4 n / 2 \times n / 2$ sub-meshes (in this case, the submeshes are quadrants). Our algorithm consists of 3 phases. In the first phase, packets move either 0 or $n / 2$ steps in their column so as to reach their virtual source in their intermediate sub-mesh. After this phase each processor contains 0,1 , or 2 packets. During the second phase each packet is routed within its sub-mesh to its 
virtual destination. Any processor is now the intermediate destination of 0,1 or 2 packets. It is easy to see that the $(2,2)$ mapping thus defined can be completed to form 2 permutations which may be routed off-line using lemma 3 . During the last phase, packets travel either 0 or $n / 2$ steps to their final destination. The storage requirements of the algorithm are the same as those of the algorithm for lemma 3 .

Theorem 2 On an $n \times n$ mesh, any permutation can be off-line routed in $2.25 n+3$ steps with at most 4 packets per processor per time step.

Proof. The proof is analogous to theorem 1. Divide the mesh into $16 n / 4 \times n / 4$ sub-meshes with the sub-meshes forming a $4 \times 4$ mesh. First we send each packet to its virtual source by moving $0, n / 4, n / 2$ or $3 n / 4$ steps in the column. The second phase consists of sending each packet from its virtual source to its virtual destination, which is a $(4,4)$ mapping, realized using lemma 4 . During the last phase, packets must travel $0, n / 4, n / 2$ or $3 n / 4$ steps to their actual destination. By sending those that must go farthest first, this can be achieved in $3 n / 4$ steps. The storage requirements are the same as those of the algorithm in lemma 4.

We are now ready to present our $2.2 n+5$ step algorithm to route permutations in an off-line manner. Our algorithm will require queues of size at most 14.

Theorem 3 On an $n \times n$ mesh, any permutation can be routed off-line in $2.2 n+5$ steps with at most 14 packets at any processor in a given time step.

Proof. Divide the mesh up into $25 n / 5 \times n / 5$ sub-meshes, considering the submeshes to form a $5 \times 5$ mesh, which we will refer to as the super-mesh.

Our first approach might be to use the following three phase algorithm: In the first phase, each packet travels either $0, n / 5,2 n / 5,3 n / 5$, or $4 n / 5$ steps using only column edges in order to get to its virtual source (in its intermediate sub-mesh). The second phase now consists of a $(5,5)$ mapping, where each packet is routed from its virtual source to its virtual destination. In the last phase each packet travels to its actual destination, using only row edges. The problem is that we do not know how to do the $(5,5)$ mapping efficiently.

Instead, we will do the following: Packets for which the actual source (destination) is the same as the virtual source (destination) are called short-distance packets and will not participate in the above three-phase algorithm. The remaining packets are long-distance packets, and these will perform the algorithm outlined above. This means that at most 4 long-distance packets could come to any virtual source, and at most 4 packets wish to go to any virtual destination in the intermediate routing stage. Each sub-mesh has to solve a $(4,4)$ mapping, and this can be done in $3 n / 5+3$ steps using lemma 4. Routing the long-distance packets therefore takes a total of $2.2 n+3$ steps, and the storage requirements are as specified in lemma 4 . 


\begin{tabular}{l|l|l|l|l|l|}
\multicolumn{1}{c}{} & \multicolumn{1}{c}{2} & 2 & 3 & 4 & 5 \\
\cline { 2 - 6 } 1 & 3 & 1 & 1 & $\mathrm{x}$ & 3 \\
\hline & 2 & 1 & 1 & 2 & $\mathrm{x}$ \\
\cline { 2 - 6 } 3 & $\mathrm{x}$ & 4 & 5 & 4 & 5 \\
\cline { 2 - 6 } 4 & 2 & $\mathrm{x}$ & 5 & 2 & 5 \\
\cline { 2 - 6 } 5 & 3 & 4 & $\mathrm{x}$ & 4 & 3 \\
\hline
\end{tabular}

Table 1: $(2,2)$ mapping assignments

\begin{tabular}{|c|c|c|}
\hline Source & Dest. & Solving sub-mesh \\
\hline 1 & 4 & 3 \\
\hline 2 & 5 & 4 \\
\hline 3 & 1 & 1 \\
\hline 4 & 2 & 2 \\
\hline 5 & 3 & 5 \\
\hline
\end{tabular}

Table 2: $(1,1)$ mapping assignments

We now have to route the short-distance packets. Each sub-mesh contributes 9 routing sub-problems, that involve only short-distance packets. Of these, 5 subproblems consist of packets whose virtual destination is the same as their actual destination. These are called column routing problems. Five problems consist of packets that are already at their virtual source. We will call these row routing problems. The plan now is to solve the column routing problems somewhere along the way in the column, rather than at the intermediate sub-mesh, and similarly with the row routing problems.

Consider the 5 sub-meshes in a row (column) of the $5 \times 5$ super-mesh. There are 25 row routing sub-problems that involve packets that travel among these submeshes. We will solve these problems using a three-phase algorithm as before, but the sub-mesh that solves these problems will be defined by Table 1, and by Table 2. To distinguish them for the sub-meshes that are solving the $(4,4)$ mappings, we will call these the solving sub-meshes. Call the processor corresponding to the actual source (destination) of a packet in the solving sub-mesh the acting source (destination).

The algorithm for the short-distance packets is now clear: Move to the acting source, then to the acting destination (via 3 permutations in the solving sub-mesh), and then to the actual destination. Therefore, each solving sub-mesh has to solve a $(2,2)$ mapping in the intermediate phase, as well as a $(1,1)$ mapping. Note that 
the assignments of the solving sub-meshes from Tables 1 and 2 have the following property:

Lemma 6 A packet has to travel at most $2 n / 5$ steps from its actual source to its acting source, and at most $2 n / 5$ steps from its acting destination to its actual destination.

Proof. Obvious from Tables 1 and 2.

We can therefore divide the set of all routing problems as those solved in the intermediate sub-meshes as part of $(4,4)$ mappings, those solved in the solving submeshes as part of $(2,2)$ mappings and $(1,1)$ mappings. We need to show that all these problems can be solved simultaneously in the requisite time with the claimed bound on the queue size.

Lemma 7 Every packet in a column routing problem can move to its acting source and perform a column permutation in $3 n / 5$ steps, at the same time as long-distance packets are moving to their virtual sources, in such a way that no collisions ever occur.

Let packet $p$ travel to its acting source, and then travel to processor $d$ as a result of the column permutation. Processor $d$ is in the same column as p's source, and is in its solving sub-mesh. In order to avoid conflict with the long-distance packets that are moving to their virtual sources, we do the following: packet $p$ simply travels straight in the column to $d$. Since $d$ can be at most $n / 5$ away from $p$ 's acting source, this takes at most $3 n / 5$ steps.

We now make the following useful observation. Consider a packet $p$ in a column routing problem. If the three-phase permutation routing between its acting source to its acting destination is performed in a column-row-column manner, then the last phase is in some sense, redundant. This is hecause at the end of two phases (column and then row permutations), it is in the same column as its acting destination, which is in the same column as its actual destination. That is, after 2 phases of the routing algorithm, such a packet can simply move directly to its actual destination. Similarly if row routing problems are solved in a row-column-row manner in their solving sub-meshes, then the last row permutation phase can be omitted. As we will see later, this observation will help us route long-distance packets from their virtual destination to their final destination simultaneously with routing row routing problems.

We go on to describe the schedule for the packets; we will prove the bounds on the time and queue size later. 


\section{Algorithm 1: Off-line routing on an $n \times n$ mesh}

Phase 1 (3n/5 steps):

- Long-distance packets move using only column edges to the virtual source from the actual source.

-Column routing problems move (using column edges) to the solving sub-mesh, and perform a permutation in the column.

-Row routing problems move in the rows to the solving sub-mesh, and perform a permutation in the row.

Phase 2 (n/5 + 1 steps):

-Long-distance packets continue to move towards their virtual sources.

-Column routing problems that are part of the $(2,2)$ mappings perform a row permutation.

-The row routing problems that are part of $(1,1)$ mappings perform a column permutation. All other packets remain stationary.

Phase 3 (3n/5 + 3 steps):

-Long distance packets start routing themselves from their virtual source to their virtual destination.

-All other packets remain stationary.

Phase 4 (n/5 + 1 steps):

-Column routing packets that are part of $(1,1)$ mappings perform a row permutation.

-Row routing packets that are part of $(2,2)$ mappings perform a column permutation.

-All other packets start moving towards their actual destination.

Phase 5 (3n/5 steps):

-All packets move towards their actual destination.

end

Lemma 8 The following conditions hold at the end of Phase 1: (1) Long-distance packets are 0 or $n / 5$ steps away from their virtual sources. (2) Short distance problems have all moved to their solving sub-meshes, and performed the first phase of the three-permutation intermediate routing.

Proof. Notice that every packet in a short-distance column routing problem can move to its acting source and perform a column permutation in $3 n / 5$ steps, at the same time as long-distance packets are moving to their virtual sources, in such a way 
that no collisions ever occur. To achieve this, let packet $p$ travel to its acting source, and then travel to processor $d$ as a result of the column permutation. Processor $d$ is in the same column as p's source, and is in its solving sub-mesh. In order to avoid conflict with the long-distance packets that are moving to their virtual sources, we do the following: packet $p$ simply travels straight in the column to $d$. Since $d$ can be at most $n / 5$ away from $p$ 's acting source, this takes at most $3 n / 5$ steps. The short-distance packets that are part of row routing problems move using only row edges, and clearly do not interfere with either long-distance packets or with the column routing problems. Therefore, (2) holds for all short-distance packets. Since no collisions occur, no long-distance packet is ever delayed. The distance between the actual source and the virtual source for any packet is at most $4 n / 5$, and therefore we have (1).

Lemma 9 At the end of Phase 2, (1) Long-distance packets are at their virtual sources. (2) Column routing problems that are part of $(2,2)$ mappings and row routing problems that are part of $(1,1)$ mappings are done with two phases of the three-permutation intermediate routing phase.

Proof. The packets from the $(2,2)$ column routing problems route two permutations in the rows (using lemma 2) in $n / 5+1$ steps. Since these use only row edges, they do not collide with the other packets. Each long-distance packet moving in its column towards its virtual source is either 0 or $n / 5$ steps away from its virtual source. Using lemma 5 , we can perform a column permutation inside each sub-column (the portion of a column inside a sub-mesh) at the same time as moving long-distance packets $n / 5$ away in $n / 5$ steps.

Lemma 10 At the end of Phase 3, all the long-distance packets are at their virtual destinations.

Proof. The proof is by using lemma 4.

Lemma 11 At the end of Phase 4, (1) Each packet can get to its destination using either row or column edges, but never both. (2) Each packet is less than $3 n / 5$ steps from its destination.

Proof. Once again, the row routing problems participating in the $(2,2)$ mappings do not affect the other packets. We will route the long-distance packets in the rows on a strictly need-based basis that is, each packet will leave only when it has to. This means that the only long-distance packets that move in Phase 4 are the ones in the sub-meshes that form the outside edges of super-mesh. This implies that packets are moving out of those sub-meshes, but not into them, and no other sub-meshes have packets moving out of them. Therefore, we can apply an analog of lemma 5 , and so we can route both the long-distance packets and perform one 
permutation in $n / 5$ steps. Since the long-distance packets that were $4 n / 5$ steps away moved without being delayed during this phase, all long-distance packets are at most $3 n / 5$ steps away from their actual destinations. The row routing packets that are part of $(2,2)$ mappings perform two column permutations as in lemma 2 in $n / 5+1$ steps.

All packets in column routing problems have performed a column and a row permutation in their solving sub-mesh. This means that each such packet can move to its acting destination via a column permutation in its solving sub-mesh. This proves (1) and lemma 6 implies (2) for such packets. Row routing packets can be similarly analyzed.

Lemma 12 At the end of Phase 5, all packets have reached their actual destinations.

Proof. Route each packet at the last possible instant. Since we are routing a permutation, and no packets need to turn, there will never be any collisions.

Lemma 13 The queue size in this algorithm does not exceed 14.

Proof. The queue size at the end of the first $4 n / 5$ steps is an upper bound on the size of the queues during Phases 1 and 2, because the only steps in which the queue at a processor can grow is when it receives a packet for which it is the virtual or acting source. From lemma 1 , we know that a single permutation within a sub-mesh can be performed with 1 packet at every node, two permutations can be performed with 2 packets at every node (by lemma 2), and finally, four permutations can be performed with 4 packets at every node (by lemma 4 ). In other words, performing permutations within sub-meshes does not increase the size of the queues. Each processor can be the virtual source for at most 4 packets and the acting source for at most 6 packets (corresponding to row and column $(2,2)$ and $(1,1)$ mappings). This implies that the maximum queue size until the end of Phase 3 is 10 . Recall that we use a "need-based" strategy for the remaining steps. In the worst case, a processor can receive packets from all four of its neighbors in the same time step, but not have started routing any of the 10 packets stationed at it at the end of Phase 3 (except for permutations within the sub-mesh). This is the worst case, because a processor must get rid of any such new packets that arrived at it, owing to the need-based strategy. The queue-size is therefore, bounded by 14 packets.

Lemmas $8,9,10,11,12$, and 13 prove our theorem.

\section{Discussion}

The algorithms described in this paper are simple conceptually, and use very small queues. We would like to investigate how far these techniques can be pushed. In particular, two questions seem worth answering. Is it possible to generalize this approach in order to obtain a family of algorithms that exhibit a trade-off between 
time and queue-size? Secondly, can this approach be used to obtain a $2 n-2$ step off-line routing algorithm that uses very small queues (say of size less than 10)?

We are interested in the possibility of an algorithm that recursively solves the "corner problems" somewhere in the middle of the mesh, while routing all the other packets using the approach outlined in this paper. The existence of an on-line algorithm to route permutations using very small queues remains an interesting open problem. Often in practical settings, packets do not travel very far; indeed, the distance traveled by any packet is bounded. The problem of routing permutations with locality is one for which we are interested in finding either an off-line or an on-line solution.

Multi-packet routing is another problem where some of these ideas may find an application. It would also be interesting to see if these algorithms could be extended $\mathrm{n}$ the torus, and other networks.

\section{References}

[AB90] F. Annexstein and M. Baumslag. A unified approach to off-line permutation routing on parallel networks. In Symposium on Parallel Algorithms and Architecture, pages 398-406. ACM, 1990.

[Kun88] M. Kunde. Routing and sorting on mesh-connected arrays. In Aegean Workshop on Computing: VLSI algorithms and architectures. Vol.319 of Lecture Notes in Computer Science, Springer Verlag, NY, pages 423-433, 1988.

[LMT89] T. Leighton, F. Makedon, and I. Tollis. A 2n-2 step algorithm for routing in an $\mathrm{n} \times \mathrm{n}$ array with constant size queues. In Symposium on Parallel Algorithms and Architecture, pages 328-335, 1989.

[R091] S. Rajasekaran and Overholt. Constant queue routing on a mesh. In Symposium on Theoretical Aspects of Computer Science, pages 444-455, 1991.

[TK77] C. Thompson and H. Kung. Sorting on a mesh connected parallel computer. Communications of the ACM, 20:263-270, 1977. 